\title{
Yang Mills short distance potential and perturbation theory
}

Nikolai Husung ${ }^{a}$, Alessandro $\mathrm{Nada}^{a}$ and Rainer Sommer ${ }^{* a, b}$

${ }^{a}$ John von Neumann Institute for Computing (NIC), DESY, Platanenallee 6, 15738 Zeuthen, Germany

${ }^{b}$ Institut für Physik, Humboldt-Universität zu Berlin, Newtonstr. 15, 12489 Berlin, Germany

E-mail: nikolai.husung@desy.de, alessandro.nada@desy.de,

rainer.sommerddesy. de

We compute the coupling $\alpha_{\mathrm{qq}}$ defined in terms of the static quark force by simulating the $\mathrm{SU}(3)$ Yang-Mills theory at lattice spacings down to $10^{-2} \mathrm{fm}$, keeping the volume large. In order to systematically improve the approach to the continuum, we subtract the leading cutoff effects in Symanzik's effective theory, resumming the leading $\log (a / r)$-term by renormalization group improvement. Subsequently we extrapolate with $\bar{g}^{2}\left(a^{-1}\right)^{\hat{\gamma}_{1}}(a / r)^{2}$ corrections to the continuum limit. We finally investigate the applicability of continuum perturbation theory, extract the puregauge $\Lambda$-parameter at different values of $\alpha_{\mathrm{qq}}$ and different orders of perturbation theory and compare to other methods.

DESY 20-012

37th International Symposium on Lattice Field Theory - Lattice2019

16-22 June 2019

Wuhan, China

${ }^{*}$ Speaker. 


\section{Introduction}

Since a few years the most precise and reliable determinations of the strong coupling arise from low-energy experiments combined with lattice gauge theory. The determined value,

$$
\alpha_{\mathrm{MS}}^{(5)}\left(M_{Z}\right)=0.1182(8) \quad \text { FLAG } 19 \text { (lattice) [1], }
$$

agrees well with the phenomenological/perturbative analysis of experimental results at larger energy scales evolved to $M_{Z}$,

$$
\alpha_{\mathrm{MS}}^{(5)}\left(M_{Z}\right)=0.1174(16) \quad \text { PDG } 16 \text { (non-lattice) [2] }
$$

Bazavov et al. used the short-distance potential computed on the lattice and matched to perturbation theory [3]. The cited result,

$$
\alpha_{\overline{\mathrm{MS}}}^{(5)}\left(M_{Z}\right)=0.1166(10)
$$

enters the average in (1.1), but is not in too good agreement with it. This motivates a precision study of the short-distance potential and its comparison to perturbation theory. In general, if we want to reduce the error of $\alpha_{\overline{\mathrm{MS}}}$ further, we need to understand perturbative errors better [4].

\section{$1.1 \alpha_{\overline{\mathrm{MS}}}$ from the static potential}

The potential is of particular interest because its perturbative expansion is known to a high order of perturbation theory (see [5] and references therein). Counting the leading one-gluon exchange potential as no loop, the accuracy is three loops.

Unfortunately there are also some caveats. First, the static potential is infrared divergent, starting at three loops [6]. It is known how to re-sum the divergent graphs, but the final expression involves an enhanced $\log (\alpha) \alpha^{4}$-term on top of the $\alpha^{4}$ one; moreover there is a remaining infrared sensitivity hidden in the choice of the "ultra-soft" scale made in the resummation based on $\mathrm{pN}$ RQCD [5]. Second, the potential is defined from the large- $t$ behavior of Wilson loops, $W(r, t)$, and the limit needs to be controlled. Third, since only the perturbative expansion of the large-volume potential is known, one needs very large lattices in order to reach small distances at a few lattice spacings. The third point can be avoided by finite-volume couplings and a step-scaling strategy [7].

To be more specific about the second point, we consider the coupling

$$
\bar{g}_{\mathrm{qq}}^{2}(1 / r)=3 \pi r^{2} F(r), \quad F(r)=\frac{\mathrm{d}}{\mathrm{d} r} V(r) .
$$

and its $\beta$-function, $\beta_{\mathrm{qq}}\left(\bar{g}_{\mathrm{qq}}(\mu)\right)=\mu \frac{\mathrm{d}}{\mathrm{d} \mu} \bar{g}_{\mathrm{qq}}(\mu)$ in the form

$$
\begin{aligned}
\beta_{\mathrm{qq}}\left(\bar{g}_{\mathrm{qq}}\right) & =-\bar{g}_{\mathrm{qq}}^{3}\left[b_{0}+b_{1} \bar{g}_{\mathrm{qq}}^{2}+b_{2} \bar{g}_{\mathrm{qq}}^{4}+\left(b_{3}+b_{3 \mathrm{~L}} l\right) \bar{g}_{\mathrm{qq}}^{6}+\left(b_{4}+b_{4 \mathrm{~L}} l+b_{4 \mathrm{LL}} l^{2}\right) \bar{g}_{\mathrm{qq}}^{8}+\ldots\right] \\
b_{2}(4 \pi)^{3} & =1.6524, b_{3}(4 \pi)^{4}=4.9449, b_{3 \mathrm{~L}}(4 \pi)^{4}=1.2538 \\
b_{4 \mathrm{~L}}(4 \pi)^{5} & =9.8088, b_{4 \mathrm{LL}}(4 \pi)^{5}=-1.6463, \quad l=\log \left(3 \bar{g}_{\mathrm{qq}}^{2} /(8 \pi)\right) .
\end{aligned}
$$

The coefficients $b_{i}$ are evaluated for $N_{\mathrm{f}}=0$ and $b_{4}$ is not known. $\beta_{\mathrm{qq}}$ is obtained from the perturbative expansion of the static force (including the so-called soft and ultra-soft contributions) and setting the ultra-soft scale to the recommended value $\mu_{\mathrm{US}}=\mu \times 3 \bar{g}_{\mathrm{qq}}^{2} /(8 \pi)$ [5]. The scale of the coupling is $\mu=1 / r$. In the above formulae, factors of $4 \pi$ are arranged such that we see coefficients of $\alpha_{\mathrm{qq}}=\bar{g}_{\mathrm{qq}}^{2} /(4 \pi)$ inside the bracket. The $b_{3}$ and $b_{4 \mathrm{~L}}$ terms give significant contributions for a typical range $0.2 \leq \alpha_{\mathrm{qq}} \leq 0.3$. 


\subsection{Yang-Mills theory}

Understanding the above pros and cons may be possible in a simplified setting, namely the pure gauge theory, where we can afford to simulate very small lattice spacings. Furthermore, recent determinations of the $\Lambda$-parameter in the $N_{f}=0$ theory,

$$
\begin{aligned}
& \sqrt{8 t_{0}} \Lambda \frac{(0)}{\mathrm{MS}}=0.6227(98) \quad[8] \\
& \sqrt{8 t_{0}} \Lambda \frac{(0)}{\mathrm{MS}}=0.5968(33) \leftarrow w_{0} \Lambda_{\overline{\mathrm{MS}}}=0.2154(12) \quad[9] .
\end{aligned}
$$

are not in agreement (we converted from $w_{0}$-units to $t_{0}$ units with the help of our simulations described below) [10]. While there is little doubt about the computation of [8], since $\alpha \approx 0.1$ was reached by non-perturbative step-scaling, a confirmation/improvement of (1.8) would be welcome, as the pure gauge theory result enters the decoupling strategy for renormalization problems [11]. For these reasons we study again the pure gauge theory and consider the extraction of $\alpha$ from the short-distance potential.

\section{Simulations}

We simulated lattices with $L \approx 4 r_{0} \approx 2 \mathrm{fm}$ and resolutions $L / a$ ranging from 32 to 192 . The smallest lattice spacing is $\approx 10^{-2} \mathrm{fm}$. In comparison to [3] we reach a factor $\approx 1 / 15$ further down in the relevant variable $a^{2}$. However, we use a standard Wilson action, in comparison to the treelevel Symanzik improved action of [3]. The smallest lattice spacing is new in comparison to the results presented in [12]. Topology freezing is avoided by open boundaries in time [13].

One reason why we opted against Symanzik improvement is that we need the limit

$$
V(r)=\frac{1}{a} \lim _{t \rightarrow \infty} \log (W(r, t) / W(r, t+a)) .
$$

Unfortunately, even in the pure gauge theory, where we employ the one-link integral [14] for the time-like links, the signal-to-noise ratio grows rapidly with $t$ : thus, both the smearing of the spatial links of the Wilson loops and the GEVP $[15,16]$ are crucial in obtaining a reliable result. The latter method is based on correlation functions with a positive spectral representation. Since violations of positivity are quite strong for improved actions [17, 18], we avoid their use. In fig.1 we demonstrate a typical case for the standard Wilson action. Extracting the ground state potential is not trivial at large $r / a$, even when strict positivity gives a rigorous mathematical basis for the GEVP method.

\section{Strategy}

Our strategy is similar to [12]. We eliminate the unphysical self-energy of the static potential, consider the force and analyse it in terms of the physical (regularisation-independent) coupling $\bar{g}_{\mathrm{qq}}$, see (1.4). From this coupling we evaluate the $\Lambda$-parameter, by integrating the renormalization group equation exactly, after truncating the $\beta$-function at a given order. The perturbative uncertainty in $\Lambda$ due to the missing $b_{4}$ is a relative error $\sim \alpha_{\mathrm{qq}}^{3}$. However, since we are dealing with an asymptotic series, the more reliable error estimate is the effect of the last known term, $\Delta \beta_{\mathrm{qq}}=-\left(b_{3}+b_{3 \mathrm{~L}} l\right) \bar{g}_{\mathrm{qq}}^{9}$, i.e. the difference between three-loop and four-loop running. 

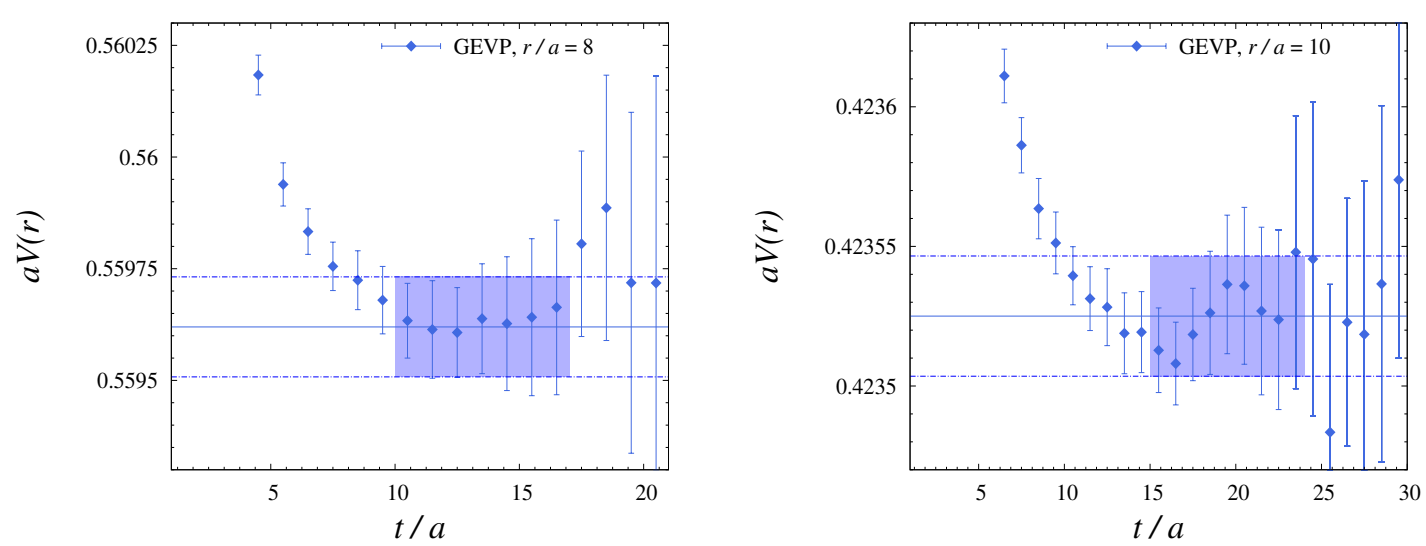

Figure 1: Extraction of $V(r)$. On the left for $a=0.03 \mathrm{fm}$ and $r=0.24 \mathrm{fm}(3 \times 3$ GEVP). On the right for $a=0.01 \mathrm{fm}$ and $r=0.1 \mathrm{fm}(2 \times 2$ GEVP). The smaller time needed in the GEVP is fixed to $4 a$ on the left and to $5 a$ on the right.

We set the scale by our own determination of $t_{0}[19]$ and evaluate $\bar{g}_{\mathrm{qq}}$ at $r / \sqrt{8 t_{0}}=0.4, \ldots, 0.25$. At $r_{\text {switch }}=0.25 \sqrt{8 t_{0}}$ we switch to using the rather precise large-volume step scaling [12] and compute

$$
\alpha_{\mathrm{qq}}\left(\left(s^{n} r_{\text {switch }}\right)^{-1}\right)=\sigma\left(\alpha_{\mathrm{qq}}\left(\left(s^{n-1} r_{\text {switch }}\right)^{-1}, s\right), \quad s=3 / 4 .\right.
$$

Apart from the plateau determinations as in fig. 1, the critical points of the computation are the continuum extrapolations $\sigma=\lim _{a \rightarrow 0} \Sigma$ of the lattice step-scaling function

$$
\Sigma(\eta, s, a / r)=\left.\alpha_{\mathrm{qq}}(1 /(s r), a /(s r))\right|_{\alpha_{\mathrm{qq}}(1 / r, a / r)=\eta} .
$$

and the truncation of the perturbative series.

\section{Continuum extrapolations}

The standard definition of the force $F(r)$ from the on-axis lattice potential $V(r)$ is

$$
F_{\mathrm{n}}(r)=\frac{1}{a}[V(r+a / 2)-V(r-a / 2)]
$$

for $r=\left(n_{\mathrm{r}}+\frac{1}{2}\right) a, n_{\mathrm{r}}=2,3, \ldots$ In [12] and in many other works, tree-level improvement is implemented before continuum extrapolations. This, however, does not account for the logarithmic corrections of the form $\left[\bar{g}^{2}(1 / a)\right]^{\gamma} a^{2} / r^{2}$ to the static potential, which are due to the anomalous dimensions of the dimension-six fields in Symanzik's effective theory [20].

Since the continuum extrapolation is crucial, we want to treat the leading $\mathrm{O}\left(a^{2}\right)$ scaling violations as accurately as possible. We thus modify naive tree-level improvement by renormalization group improvement, resumming the $\log (a / r)$ terms [20]. We start by improving the discrete derivative (4.1) in the form

$$
F_{\text {impr }}(r)=F_{\mathrm{n}}(r)-\frac{1}{24}\left[F_{\mathrm{n}}(r+a)-2 F_{\mathrm{n}}(r)+F_{\mathrm{n}}(r-a)\right] .
$$



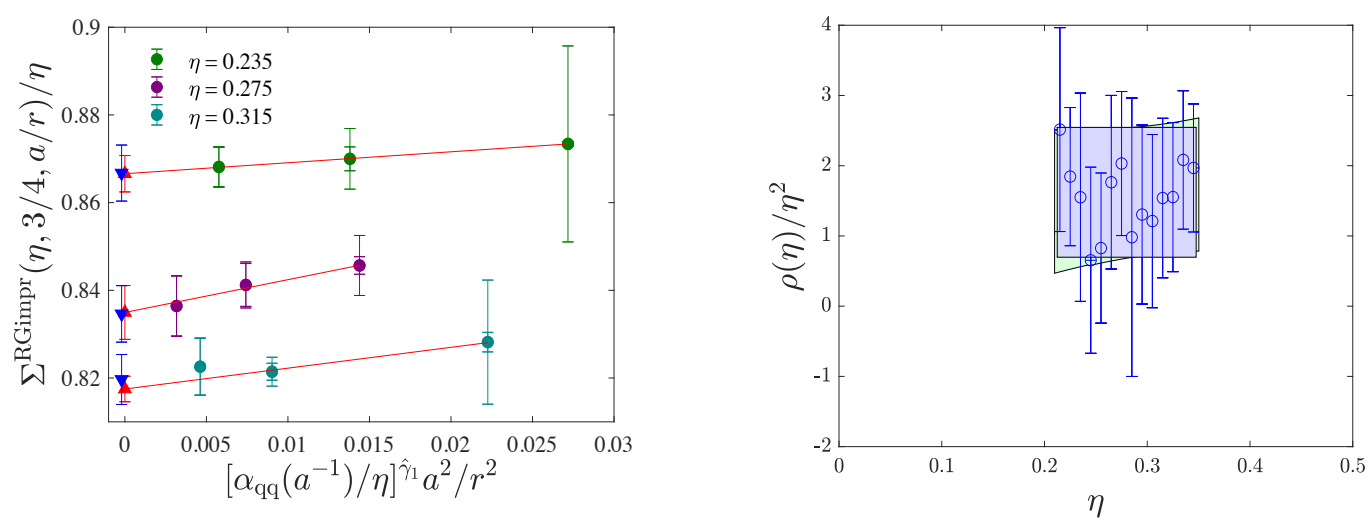

Figure 2: Left: Continuum extrapolations (4.8) at different $\eta_{i}$ : upward pointing triangles and smaller errorbars have the $B a^{4} / r^{4}$ term (see text) turned off. Right: resulting slopes $\rho_{i}$ and the fits (4.9) with one (blue band) and three free parameters (green band).

The improved $F_{\text {impr }}(r)$ is accurate up to $\mathrm{O}\left(a^{4}\right)$ except for $\mathrm{O}\left(a^{2}\right)$ effects which originate from $V(r)$ itself. The latter are now known in the form [20]

$$
\begin{aligned}
\alpha_{\mathrm{qq}}(1 / r, a / r)-\alpha_{\mathrm{qq}}(1 / r, 0)= & \alpha_{s}\left(r^{-1}\right) \frac{a^{2}}{r^{2}} \times\left\{\left[\frac{\alpha_{s}\left(a^{-1}\right)}{\alpha_{s}\left(r^{-1}\right)}\right]^{\hat{\gamma}_{1}} A_{1}(r)+\left[\frac{\alpha_{s}\left(a^{-1}\right)}{\alpha_{s}\left(r^{-1}\right)}\right]^{\hat{\gamma}_{2}} A_{2}(r)\right\} \\
& \times\left(1+\mathrm{O}\left(\alpha_{s}\left(a^{-1}\right)\right), \quad \hat{\gamma}_{1}=\frac{7}{11} \approx 0.636, \quad \hat{\gamma}_{2}=\frac{63}{55} \approx 1.145 .\right.
\end{aligned}
$$

Here we have written the expansion in terms of the renormalised coupling $\alpha_{s}$ in a scheme $s$ which is irrelevant at the considered order. The functions $A_{i}(r)$ are (RGI) matrix elements of the $d=6$ operators in Symanzik's effective action. At short distances they can be expanded

$$
A_{i}(r)=A_{i, 0}+A_{i, 1} \alpha_{s}(1 / r)+A_{i, 2} \alpha_{s}(1 / r)^{2}+\ldots
$$

and for our Wilson action we have

$$
A_{1,0}=0, \quad A_{2,0}=\frac{3}{4} .
$$

At small lattice spacing, the first term in the curly bracket in (4.3) dominates, but the second one is suppressed by one power less of $\alpha\left(r^{-1}\right)$ at small $r$.

We therefore also use the known $A_{2,0}$ and define

$$
\alpha_{\mathrm{qq}}^{\mathrm{RGimpr}}=\frac{\alpha_{\mathrm{qq}}}{1+A_{2,0}\left[\frac{\alpha_{\mathrm{qq}}\left(a^{-1}\right)}{\alpha_{\mathrm{qq}}\left(r^{-1}\right)}\right]^{\hat{\gamma}_{2}} \frac{a^{2}}{r^{2}}},
$$

where the coupling at the cutoff $\alpha_{\mathrm{qq}}\left(a^{-1}\right)$ is obtained from the measured $\alpha_{\mathrm{qq}}\left(r^{-1}\right)=\frac{3}{4} F_{\mathrm{n}}(r) r^{2}$ at $r=$ $2.5 a$ by four-loop evolution (here we use the unimproved force $F_{\mathrm{n}}$ ). Discretisation errors of $\alpha_{\mathrm{qq}}^{\text {RGimpr }}$ are then modelled as $\frac{a^{2}}{r^{2}}\left[\frac{\alpha_{s}\left(a^{-1}\right)}{\alpha_{s}\left(r^{-1}\right)}\right]^{\hat{\gamma}_{1}} \alpha_{\mathrm{qq}}\left(r^{-1}\right) A_{1}(r)$, with $A_{1}(r)=\mathrm{O}\left(\alpha_{\mathrm{qq}}\left(r^{-1}\right)\right)$. The evaluation of $\Sigma$ requires $\alpha_{\mathrm{qq}}$ as a continuum function of $r$, which is easily obtained by a local interpolation. The 
continuum limit $\sigma(\eta, s)$ is reached, asymptotically, as

$$
\Sigma^{\mathrm{RGimpr}}(\eta, s, a / r)=\sigma(\eta, s)+\rho(\eta)\left[\frac{\alpha_{\mathrm{qq}}\left(a^{-1}\right)}{\eta}\right]^{\hat{\gamma}_{1}} \frac{a^{2}}{r^{2}}, \quad \rho(\eta)=\mathrm{O}\left(\eta^{2}\right) .
$$

We assume that form for the continuum extrapolations, which we split into a three-step procedure.

1. We choose values $\eta_{i}$ covering the accessible range of $\eta$ with rather small separation; in practice we have $0.215 \leq \eta_{i} \leq 0.345$, separated by $\eta_{i+1}-\eta_{i}=0.01$. At these values we fit (fig. 2)

$$
\Sigma^{\mathrm{RGimpr}}\left(\eta_{i}, s, a / r\right)=\sigma_{i}+\rho_{i}\left[\frac{\alpha_{\mathrm{qq}}\left(a^{-1}\right)}{\eta_{i}}\right]^{\hat{\gamma}_{1}} \frac{a^{2}}{r^{2}} .
$$

Of course, we should restrict the fits to data with reasonably small lattice spacings. In practice, an exclusion of the potential at the cutoff $V(a)$ implies $r / a \geq 3.5$, which is enough for our extrapolations (see also below).

2. We then test that the slopes $\rho_{i}$ are compatible with the expected form,

$$
\rho_{i}=\eta_{i}^{2} \times\left(\rho^{(1)}+\rho^{(2)} \eta_{i}+\ldots\right),
$$

fitting to this form with parameters $\rho^{(j)}$, see fig. 2. At this point the discretization errors are known in the form

$$
\Sigma(\eta, s, a / r)=\sigma(\eta, s)+\left[\frac{\alpha_{\mathrm{qq}}\left(a^{-1}\right)}{\eta}\right]^{\hat{\gamma}_{1}} \frac{a^{2}}{r^{2}}\left(\rho^{(1)} \eta^{2}+\rho^{(2)} \eta^{3}+\ldots\right) .
$$

3. This formula, with the determined parameters $\rho^{(1)}, \rho^{(2)}$, is now used to extract the stepscaling functions at the desired points $\alpha\left(\left(s^{k} r_{\text {start }}\right)^{-1}\right), k=1,2, \ldots$

\section{Systematic errors}

The above procedure is based on a number of assumptions. We assume that after the renormalization group improved removal of the leading (in the expansion (4.6) ) $a^{2}$ errors, the left over ones are reasonably modeled by the leading term in (4.3). Deviations are higher orders in $\alpha_{\mathrm{qq}}\left(a^{-1}\right)$ which do not vary so much with $a$. They are thus expected to be effectively taken into account in the fit (4.8). However, we also discard $a^{4}$ terms in that fit. We try to estimate their effect in our errors, by adding a term $B a^{4} / r^{4}$ to the force with a coefficient $B=0 \pm 3.3$, where the \pm 3.3 error is the size of the $a^{4}$-term found at tree-level. The effect of $B$ is propagated into all quantities by standard, quadratic, error propagation. Note that it also reduces the weight of points with large $a / r$ in the extrapolations (4.8). It becomes unnecessary to apply a cut beyond the mentioned $r / a \geq 3.5$. The effect of $B$ is included in the above figures as well as the following results.

\section{Results and conclusions}

The step-scaling function at the mentioned points $\eta_{i}$ is shown in the left panel of fig. 3 . It is somewhat below the perturbative estimates at different orders. Despite the additional lattice spacing, error bars are larger than in the previous analysis [12], due to the additional systematic error coming from the $\mathrm{Ba}^{4} / \mathrm{r}^{4}$ term. A semi-quantitative agreement with perturbation theory is 

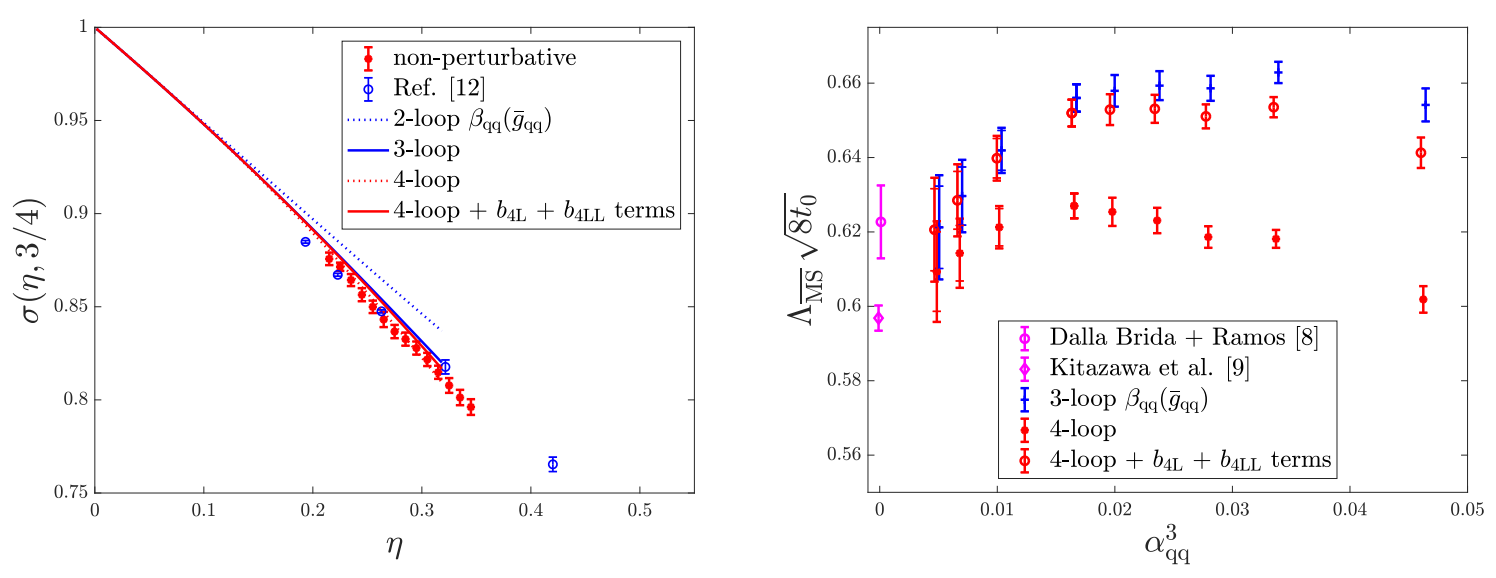

Figure 3: Left: Step scaling function compared with PT. Right: $\Lambda$-parameters determined at various values of $\alpha_{\mathrm{qq}}$ together with different orders of PT.

found over quite a large range of $\alpha$ at the level of 5-10\% in $\Lambda$, as seen on the right panel of fig. 3. For central values we should concentrate on the results using the 4-loop $\beta$-function. An extrapolation of the last few points in $\alpha^{3}$ appears to agree better with the result of [9] than with [8]. However, the precision achieved is not good enough to make a real distinction.

The fact that the $b_{4 \mathrm{~L}}$ and $b_{4 \mathrm{LL}}$ terms are as big as the 4-loop contribution to the $\beta$-function in the relevant range of $\alpha_{\mathrm{qq}}$ suggests that the limitation of the asymptotic perturbative series is reached with the 4-loop $\beta$-function in most of our range, while 5-loop accuracy may help at the edge of $\alpha \approx 0.21$. With data at $\alpha \approx 0.25$ and above, the difference of numbers with 3-loop and 4-loop (or equivalently 4-loop and 4-loop $+b_{4 \mathrm{~L}}, b_{4 \mathrm{LL}}$ terms puts a bound on the precision.

On the other hand smaller values of $\alpha_{\mathrm{qq}}$ are afflicted with too large errors at present. The main reason is that our estimate of the $a^{4} / r^{4}$ uncertainty is too large compared to the very high precision required to obtain a value of $\Lambda$ below the $5 \%$ level.

Acknowlegements. We thank our colleagues in the ALPHA-collaboration for many discussions. We acknowledge funding by the H2020 program in the Europlex training network, grant agreement No. 813942. Generous computing resources were supplied by the North-German Supercomputing Alliance (HLRN, project bep00056) and by the John von Neumann Institute for Computing (NIC) at DESY, Zeuthen.

\section{References}

[1] Flavour Lattice Averaging Group collaboration, FLAG Review 2019, 1902.08191.

[2] Particle Data Group collaboration, Review of Particle Physics, Chin. Phys. C40 (2016) 100001.

[3] A. Bazavov, N. Brambilla, X. Garcia i Tormo, P. Petreczky, J. Soto and A. Vairo, Determination of $\alpha_{s}$ from the QCD static energy: An update, Phys. Rev. D90 (2014) 074038.

[4] M. Dalla Brida, P. Fritzsch, T. Korzec, A. Ramos, S. Sint and R. Sommer, Determination of the QCD $\Lambda$-parameter and the accuracy of perturbation theory at high energies, Phys. Rev. Lett. 117 (2016) 182001. 
[5] X. Garcia i Tormo, Review on the determination of $\alpha_{s}$ from the QCD static energy, Mod. Phys. Lett. A28 (2013) 1330028 [1307.2238].

[6] T. Appelquist, M. Dine and I. J. Muzinich, The Static Limit of Quantum Chromodynamics, Phys. Rev. D17 (1978) 2074.

[7] M. Lüscher, P. Weisz and U. Wolff, A Numerical method to compute the running coupling in asymptotically free theories, Nucl.Phys. B359 (1991) 221.

[8] M. Dalla Brida and A. Ramos, The gradient flow coupling at high-energy and the scale of SU(3) Yang-Mills theory, Eur. Phys. J. C79 (2019) 720.

[9] M. Kitazawa, T. Iritani, M. Asakawa, T. Hatsuda and H. Suzuki, Equation of State for SU(3) Gauge Theory via the Energy-Momentum Tensor under Gradient Flow, Phys. Rev. D94 (2016) 114512 [1610.07810].

[10] N. Husung, A. Nada and R. Sommer, in preparation .

[11] ALPHA collaboration, Non-perturbative renormalization by decoupling, 1912.06001.

[12] N. Husung, M. Koren, P. Krah and R. Sommer, SU(3) Yang Mills theory at small distances and fine lattices, EPJ Web Conf. 175 (2018) 14024 [1711.01860].

[13] M. Lüscher and S. Schaefer, Lattice QCD without topology barriers, JHEP 07 (2011) 036 [1105.4749].

[14] G. Parisi, R. Petronzio and F. Rapuano, A Measurement of the String Tension Near the Continuum Limit, Phys. Lett. 128B (1983) 418.

[15] M. Lüscher and U. Wolff, How to Calculate the Elastic Scattering Matrix in Two-dimensional Quantum Field Theories by Numerical Simulation, Nucl. Phys. B339 (1990) 222.

[16] B. Blossier, M. Della Morte, G. von Hippel, T. Mendes and R. Sommer, On the generalized eigenvalue method for energies and matrix elements in lattice field theory, JHEP 04 (2009) 094 [0902 . 1265].

[17] S. Necco, Universality and scaling behavior of RG gauge actions, Nucl. Phys. B683 (2004) 137 [hep-lat/0309017].

[18] TUMQCD collaboration, Determination of the QCD coupling from the static energy and the free energy, Phys. Rev. D100 (2019) 114511 [1907.11747].

[19] M. Lüscher, Properties and uses of the Wilson flow in lattice QCD, JHEP 08 (2010) 071 [1006.4518].

[20] N. Husung, P. Marquard and R. Sommer, Asymptotic behavior of cutoff effects in Yang-Mills theory and in Wilson's lattice QCD, 1912.08498. 